\title{
PROBLEMS OF FRICTION FORCE MEASUREMENT sciendo BETWEEN CYLINDRICAL OUTDOOR AND INTERNAL SLIDE PARTS
}

doi:10.2478/mape-2018-0003

Date of submission of the article to the Editor: 04/2018 Date of acceptance of the article by the Editor: $07 / 2018$

\author{
MAPE 2018, volume 1, issue 1, pp. 19-25
}

\author{
Dr inż. Jan Monieta \\ Maritime University of Szczecin, Poland
}

\begin{abstract}
The article presents the determination of an exemplary measure in the assessment of the technical state of sets of mechanical objects at the stage of manufacture, use and maintenance. The technical state of the assemblies is influenced by the quality of the friction-cooperating elements, the quality of basic functions, the surroundings and the diameter clearance of kinematic node components. Energy wasted to overcome the frictional resistance is a significant part of the energy supplied, which is why current friction losses are minimized.

The article includes analysis of the phenomena occurring in the cylindrical pairs in the presence of static and kinetic friction. The many factors affecting the course and values of friction force between cylindrical elements performing relative reciprocating motion have been indicated. The subject of the article is the method of measuring the friction force between a fixed and movable cylindrical element of the mechanical object. The gradual increase of the friction force component takes place by means of easily accessible force.

The results of experimental tests of the friction force in different laboratory conditions under are also included. The effect on the measured friction force was investigated: of friction elements geometry and the surface condition after manufacture and/or operation. Scatter results of static friction force depending on the geometry and surface state can be significant. Examples of surface state images are shown. A friction force model was also developed depending on factors affecting its value.
\end{abstract}

Keywords: mechanical object, guide and cylinder, measurement, friction force

\section{INTRODUCTION}

In evaluation a technical state of the teams of sets of mechanical objects at the stage of manufacture, use and maintenance take into account the working process parameters, characterizing the quality of basic functions. The energy lost to overcome the frictional resistance is a significant part of the energy supplied, especially in large work and critical machines, so is the friction losses are currently being minimized. The evolution of the ownership of the node tribological is in the phase of design and construction, the course of the manufacturing process and operation: shape and dimensions, materials, inputs, ownership of the surface layer, lubricating substances and the environment recommendations, etc.

Friction accompanies every movement, counteracting mutual movement that is why tribological systems should be shaped in such a way that they are subject to destructive influences at the friction ridges to a small extent. Friction is the result of mechanical interactions of micro-inequities and molecular interaction of friction-related elements.

The important role of pneumatic and hydraulic systems in production factory can be seen in many technical devices. With the evolution of construction and technology, speed and reliability, the characteristics of small friction and long durability are required more and more often for all pairs cylinder - pin. 


\section{CLASSIFICATION OF FRICTION CONSIDERING TO MOVEMENT}

Regardless of the type of tribological tests it is recommended that perform a quantitative measurement of resistance of friction depending on the load, the friction pairs cooperation time, temperature, etc. (Hebda, 2007). To determine the dynamic and energetic effects of friction are use (Włodarski, 1998): friction force, friction torque, coefficient of friction, the friction heat, the temperature of the friction. The state of the element's surface is determined by a set of geometrical, physicochemical and mechanical features are shaped in the production and exploitation phase, and it is determined by macroscopic, microscopic and submicroscopic errors (Monieta and Łukomski, 2005).

So far no general dry friction theory has been developed because it is dependent on the set of phenomena, parameters and friction conditions. The many studies have been carried out and many dependencies have been formulated, among others Columb expressed the formula on the friction force:

$$
T=\mu N+A
$$

where: $\mu$-friction coefficient, $N$ - thrust, $A$-amendment of frictional force depends on the molecular interactions of the surface friction.

At the interface between two bodies interact are randomly random surface irregularities by each other. The contour contact surface is the outline of the actual contact fields, and the actual contact surface $A_{r}$ is the sum field of the elementary contact surfaces that occurs at the contact points of the inequalities $A_{i}$ :

$$
A_{r}=\sum_{i=1}^{n} A_{i}
$$

At the interface, external sliding and internal friction occur in the deformed volume of the surface layer of rubbing elements. In the adhesive-mechanical model in Kragielski's theory, the force of adhesion is related to the theory of molecular interaction. The individual friction force $T_{j}$ acts on the elementary area of contact surface:

$$
T=A_{s} \sum_{i=1}^{n} T_{j i}
$$

where: $A_{s}$ - contact surface if there is the mechanical and molecular interaction.

The unitary friction force for a molecular-mechanical interaction is equal to:

$$
T_{j}=T_{j m o}+T_{j m e}
$$

where: $T_{j m o}-$ unitary friction force with molecular interaction, $T_{j m e}-$ unitary friction force at the mechanical influence.

The extent of friction in the material depends on the condition of the surface (pollution, moisture, contamination of lubricants, ambient air composition, etc.). Among the frictional resistances when two solids move relative to each other, one can distinguish between $T_{a d h}$ adhesive friction and $T_{\text {koh }}$ cohesive resistance (Hebda, 2007):

$$
T_{t}=T_{\text {adh }}+T_{\text {koh }}
$$

The external friction coefficient is difficult to measure due to the non-recurrence of the conditions. When the cylinder moves (rolls) under the influence of force $F$ it contacts the base along the surface $A B$ (Fig. 1). Turning is possible when the force $F$ exceeds the limit value (Obłąkowska-Mucha). Under the influence of force $F$, the pressure at point $B$ increases and in $A$ decreases. The point of application of force $N^{\prime}$ moves towards $F$. As the $F$ increases, the shift increases until the limit value $\mu_{t}$ is reached. There are opposite moments: $\mu_{t} \times N^{\prime}$ and $r \times$ $T$. The condition of equilibrium condition is equality: $\mu_{t} \times N^{\prime}=r \times T$, hence the coefficient of rolling friction is:

$$
\mu_{t}=\operatorname{Tr} N
$$




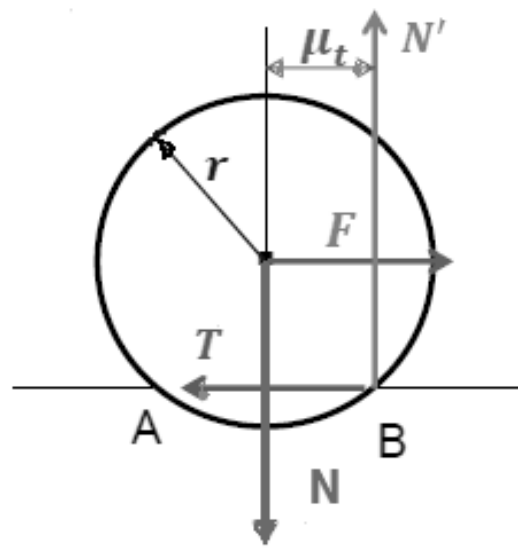

Fig. 1. Asymmetric reaction of the ground: $T-$ friction force, $F=T-$ force applied to the roller, $N$ - normal force $N=m g, N^{\prime}-$ ground reaction force

The frictional moment is balanced by size, which creates a tangential force of friction multiplied by the diameter of the rotating cylinder $d=2 r$ :

$$
M_{t}=N \mu_{c a l} d
$$

Mixed-friction is a combination of at least two types of friction: dry friction, boundary or liquid friction. The frictional force is the sum of the forces of interaction of micro-contact friction external and internal friction in microstrips of lubricant substance. The mixed friction force can be expressed as the difference between dry and liquid friction:

$$
T_{m i}=T_{d}-T_{l}=N \mu_{c a l}-\eta A \frac{d c}{d h}
$$

where: $T_{d}$-dry friction force, $T_{1}-$ liquid friction force, $\eta$-dynamic viscosity of the lubricating liquid, $c$ - slip velocity with relative motion, $h$ - oil layer thickness.

Mixed friction exists under conditions of insufficient quantity of lubricant, and when it is sufficient to fill all unevenness of both surfaces, taking into account the contact deformation.

\section{STATE OF KNOWLEDGE}

\section{Measurements of the friction force of cylindrical elements cooperating frictionally}

The tests of friction force and moment are carried out in laboratory and operating conditions. In laboratory, the real operating conditions are modelled: contact geometry, covering of friction areas, motion kinematics, load dynamics, unit pressures, lubrication, temperature, etc. Resistance to relative movement of friction elements is measured using dynamometers, using various types of electrical sensors (resistance, piezoelectric, inductive etc.) (Hebda, 2007).

Variable profile of elements obtained during production is the reason of variable values of frictional resistance which are caused by the limited (real) contact in a connection. The paper presents the test stands for the measurement of the force and moment of friction of sleeve shaft couple (Dudziak et al., 2014). The sleeve was connected with shafts which were manufactured with radial error, cross-section deviations and their compilations. The experimental investigations were performed for determine the influence of the form deviations and angular positions of the shaft on the value of axial friction force (during shaft sliding) and moment of friction (during shaft rotation).

From the literature and United States patents (Ivanaga and Kiriki, 2002; Kiriki, 2002) and it appears that at present the aim is to minimize frictional losses in internal combustion engines, which results in a reduction of fuel consumption (Holmberg et al. 2014) and (Zabelin, 2014). The increase of friction power is an important measure of wear processes occurring in tribological nodes and is a factor determining the efficiency of machines. 
Often in tribological tests it is necessary to conduct friction measurements on sections of existing elements or on very small samples of new materials or coatings of friction pair with reciprocating motion. Such tests may be carried out on the friction testing device in reciprocating motion. The construction of the station makes it possible to carry out tests in a given (variable) range of motion, loads and slip velocity, according to the tested friction node. The general principle of operation of the static friction station is based on determining the tangent of the angle of inclined plane, at which the relative motion begins between the sample of the material being tested and the friction-cooperating element mounted on the tilting arm of the (Capanidis et al., 2015). According to the basic relations between the forces occurring on an inclined plane, the angle of inclination $\alpha$ corresponds to the angle of friction, and thus the tangent of this angle corresponds to the static friction coefficient $\mu_{0}=\tan \alpha$. In the tests it is possible to determine the effect of force $F$, causing a specific unit pressure $N$, time of action $\tau_{p}$ load, surface roughness of associated materials, ambient environment, etc.

The device consists of two lying on a trolley, ball bearing track so that they can move in the same direction (Capanidis et al., 2015). Researches of friction were the designation of kinetic and static friction coefficient and wear.

Engine and lubricant designers are constantly improving the performance of internal combustions engines by reducing the power losses and emissions. The majority of the mechanical frictional losses generated in an engine can be attributed to the main tribological assembles of an engine, the valve train, piston assembly and engine bearings. The validation in work (Mufti, 2004) was achieved by experimentally characterizing the frictional losses taking place in the major tribological components of a single cylinder gasoline engine. A system was developed to evaluate experimentally, frictional losses in all the main tribological components of an engine under fired conditions.

The friction characteristics of pneumatic cylinders have not been studied sufficiently (Belforte, 2003). Both papers and manufacture's catalogues seldom show friction characteristics of cylindrical pairs, and the lack of friction models limits the design, optimization and analysis of pneumatic cylinder systems.

The stu developed a measurement system for the friction force characteristics of pneumatic cylinders (Chang et al., 2012). The dynamic characteristics of pneumatic cylinder are affected by guide seal, piston seal, grease quality and surface of cylinder bore. In seeking to improve the friction force characteristics and to clarify the friction phenomenon of the pneumatic cylinders, this study describes friction force measurement tests of pneumatic cylinder, under both dry and lubricated conditions.

\section{Friction measurement of injector nozzles}

Examples of cylindrical friction pairs: guide - cylindrical cooperating part are of injector nozzles of internal combustion engines. So far during the acceptance tests nozzles injectors checked only if the force of friction between the nozzle body and the needle is less than the force resulting from the component of the force of gravity or not, which describes literature (Piaseczny, 1992). In this way, the injector nozzle estimate two-state were evaluated (suitably or unsuitably) without taking into account the mutual position of one element in relation to the other quantitative measurement. The dispersion of the results of the friction force between the nozzle body and the needle can be significant, up to immobilization, deciding important consequences for the engine fuel leak into the oil (Mienieta and Lorek, 2008).

Patents (Farth, 2005) provides a method for determining the injector nozzle opening pressure and the system and informs that the friction that takes place in the process will be an interference variable of the determination of this pressure.

This article presents the results of the friction force investigations between the needle and the nozzle body of marine diesel engines (Monieta, 2012). Injectors made of the alloy steel of piston internal combustion engines fed with diesel oil or residual fuels were investigated. 
Nozzles were tested in the presence of fuel oil or oil with similar properties, as well as measurements of ejection time choking the nozzle at different angles of the rotation respect to the longitudinal axis of the nozzle needle in the nozzle body.

Geometrical measurements were made of precision pairs to investigate the effect of the dimensions and shape and location errors on the values of friction forces. The influence of the angular position of the needle relative to the body are investigated, and precise measurements of the diameter clearance between the frictional couple were taken to determined its effect on the value of the maximum friction force.

The value of the maximum force of friction between the nozzle body and the needle tip is influenced by the condition of mating surfaces and their mutual positioning, pollution, etc. The research aimed to determine the component of friction force and/or the time of the movement of the needle depending on the angle of the turnover of the needle in the nozzle body, clearance, kind, applied medium and masses of the needle.

An assessment of the state of knowledge in the presented scope was carried out, and to place the author's research in the space of the state of world research.

\section{EXAMPLE INVESTIGATION RESULTS}

Kinetic friction occurs when the bodies are in motion relative to each other, therefore the kinetic friction force is less than the static friction force. In the pair, the guide in the body the part of the cylindrical load acts mainly along the axis of the cylinder. Side thrusts may occur due to shape and position errors, elastic deformations and loads due to pressure of the working medium on the surfaces in the gaps between the body and the cylinder (Monieta, 2012).

Programmed laboratory and operational program tests were carried out. In the case of software operational tests, the values of the following parameters were recorded: load, speed, acceleration, reference conditions, etc. Fig. 3 presents the idea of the method of measuring the friction force of the cylindrical couple sleeve - shaft.

Programmed laboratory and operational tests were carried out. In the case of software operational tests, the values of the following parameters were recorded: load, speed, acceleration, reference conditions, etc.

In the case of static and kinetic friction in the connection of cylinder with the cylinder type of a spigot - a bushing, the influence of such factors on the friction force can be tested as: angle of inclination $\alpha$, type of associated materials $m_{a}$, average pressure (load of pair) $N$, speed of sliding and rotation $c$, working time $\tau$ or friction path, as well as geometric parameters $P_{g}$ (diameter $D$ and length of connection $l_{t}$, diametrical clearance, bushing thickness, surface roughness of cooperation, shape errors, etc.), the number of repetitions of measurements $i_{r}$. This can be written as a model of friction force:

$$
T=f\left(\alpha, m_{a}, N, c, \tau, D, I_{t}, P_{g}, i_{r}\right)
$$

Undoubtedly, enormous roles in tribological tests in terms of the friction force values are the examinations of the surface layer and the analysis of the condition of friction surfaces. Fig. 2 shows an image of longitudinal section of guide sleeve (internal cylinder). Fig. 3 shows a portion of surface of the operated of external cylinder at a magnification field of digital microscope. In the pictures you can distinguish the kinds wear that affect the value of the friction force.

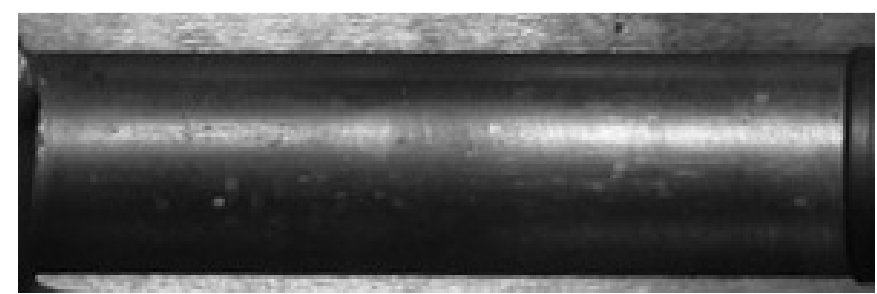

Fig. 2. Longitudinal section of the guide part of the body with the cylindrical inner surface 


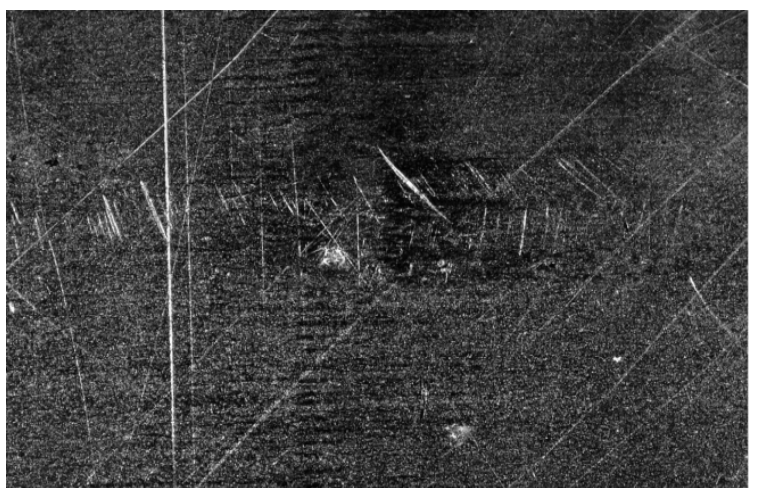

Fig. 3. A fragment of the leading part of the exploited roller at the magnification of $200 x$

Numerous experimental studies were carried out in laboratory conditions (Monieta, 2012). In works (Monieta and Lorek, 2008) the friction force courses was presented along the friction travel depending on the state of the lubricant, diametrical clearance and mutual position in longitudinal planes of friction pairs. Fig. 4 presents the results of the active experiment of the influence of the nominal diameter of the friction pair on the value of the maximum friction force for the same diametrical clearance. A non-linear relationship was obtained.

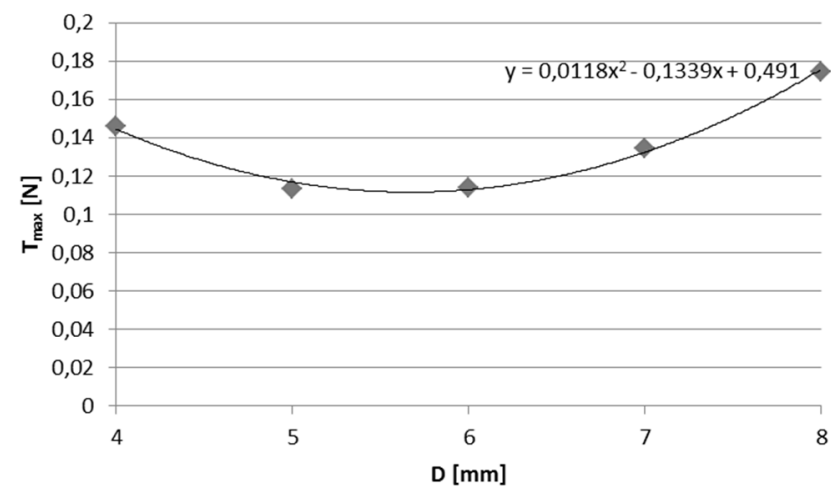

Fig. 4. Example of the influence of the nominal diameter of the friction pair on the value of the maximum friction force for the same diametrical clearance

Some of the friction pairs moved relative to each other but with a different speed, as shown in Fig. 5, where it can be seen that there is a considerable scattering of 100 exemplary realisations. Other pairs exhibited significant values of the friction force, which required the application of significant exciting forces.

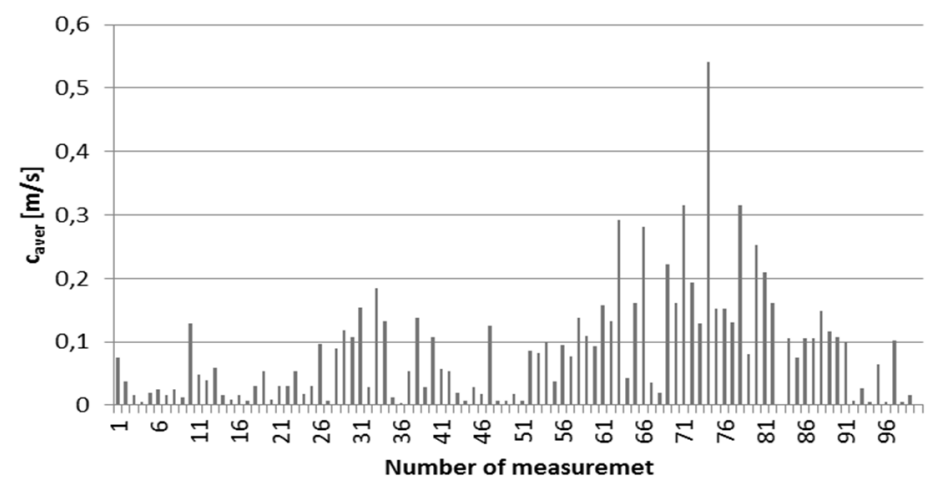

Fig. 5. Examples of average speeds of line feed of cylindrical parts from cylindrical guides at kinetic friction 
This publication presents only selected results of extensive experimental studies. There is a possibility of significant automation of tests in production conditions.

\section{CONCLUSION}

Was elaborated the measurement method of the friction force between the cylindrical outer and inner sliding surfaces. In the article was presented the experimental results to measure friction force between cylindrical sleeves and cylindrical pins. It is possible to measure of friction forces cooperating units in cylinders of different sizes and in various internal and external conditions. The results provide manufacturers a reference to design and test the produced and operated of cylindrical pairs.

The distribution of static friction results is significant depending on the geometry and surface state can be significant. These elements were tested under both dry condition and lubricated conditions.

From the measurement tests, the friction characteristics and their relationships with velocity was experimentally clarified. The speed of mutual displacement with kinetic friction is significantly different. Eventually it can be used to study friction with the purpose of testing the existing models or proposing new models.

\section{REFERENCES}

Belforte, G., Mattiazzo, G. and Mauro S. (2003). Measurement of Friction Force in Pneumatic Cylinders, Tribotest 10(1), pp. 33-48.

Capanidis, D., Kowalewski, P., Leśniewski, T., Paszkowski, M. and Wieleba W. (2015). The Role of Tribological Research in Increasing the Durability and Reliability of Machines and Devices Used in the Copper Belt. Scientific Papers of the Lower Silesian School of Business and Technology. Studies of Technical Sciences, 4, pp. 47-64.

Chang, H., Lan, C., Chen, C., Tsung, T. and Guo J. (2012). Measurement of Frictional Force Characteristics of Pneumatic Cylinders Under Dry and Lubricated Conditions. Electrical Review, 88 (7b), pp. 261-264.

Dudziak, M., Domek, Kołodziej, A., and Talaśka K. (2014). Contact Problems Between the Hub and the Shaft with a Three-Angular Shape of Cross-Section for Different Angular Positions. Procedia Engineering, 96, pp. 50-58.

Farth, A., Voigt, A. and Spickenreuther M. (2005). Method for Setting the Nozzle Opening Pressure for an Injection Nozzle and Arrangement for Carrying out the Method. 6,976,389.

Hebda, M. (2007). The Processes of Friction, Lubrication and Machine Wear. Warsaw - Radom: Institute of Sustainable Technologies.

Holmberg, K. Andersson, P. Nylund, N.O., Mäkelä, K. and Erdemir A., (2014). Global Energy Consumption Due to Friction in Trucks and Buses. Tribology International, 78, pp. 94-114.

Iwanaga, T. and Kiriki, Y. (2002). Fuel Injection Nozzle With a Member to Reduce The Frictional Force Developed Between Parts During the Clamping. 1,236,887.

Kiriki, Y. (2002). Fuel Injection Nozzle with a Member to Reduce the Frictional Force Developed Between Parts During The Clamping. 20,020,109,021.

Monieta, J. (2012). Research on the Friction Force of the Needle in the Nozzle Body of Marine Diesel Engines in the Presence of a Lubricating Medium that Account Position Changes. Tribologia, 2, pp. 41-57.

Monieta, J. and Lorek $Ł$. (2008). Researches of Friction Force of Injector Nozzles in Injector Bodies of Marine Diesel Engines in the Presence of Lubricating Compound. Journal of Polish CIMAC, 3(1), pp. 111-121.

Monieta, J., Łukomski, M. (2005). Methods and Means of Estimation of Technical State Features of the Marine Diesel Engines Injector Nozzles Type Sulzer 6AL20/24. Scientific Journals of the Maritime University of Szczecin, 5(77), pp. 383-391.

Mufti, R.A. and Priest, M. (2012). Effect of Cylinder Pressure on Engine Valve-Train Friction Under Motored and Fired Conditions. Journal of Engineering Tribology, pp. 306-314.

Piaseczny, I. (1992). Marine Repair Technology of Internal Combustion Engines. Gdansk: Maritime Publishing, pp. 394-395.

Włodarski, J.K. (1998). Exploitation of Ship Machinery. Gdynia: Gdynia Maritime University.

Zabelin, P. (2014). Internal Combustion Engine Efficiency. 0,318,507. 